\title{
Comparison of Post-Operative Nutritional Status according to the Extent of Gastrectomy and the Reconstruction Method in Patients with Gastric Cancer
}

\author{
Kyung Hwan Kim, M.D., Jong Hyuk Yoon, M.D., Geum Jong Song, M.D., Myoung Won Son, M.D., Ph.D., \\ Sung Yong Kim, M.D., Ph.D., Moo Jun Baek, M.D., Ph.D., Moon Soo Lee, M.D., Ph.D. \\ Department of Surgery, Soonchunhyang University College of Medicine, Cheonan, Korea
}

Purpose: Malnutrition is the main complication after gastrectomy and the degree may vary depending on the extent of resection. This study sought to help determine an appropriate type of resection and provide methods for addressing malnutrition after gastrectomy.

Materials and Methods: We retrospectively reviewed medical records of patients with gastric cancer who underwent radical resection at the Soonchunhyang University Cheonan Hospital between December 2012 and December 2013. A total of 100 patients were screened, and all patients were followed for 1 to 3 years. Among this group, 12 underwent total gastrectomy, 5 underwent proximal gastrectomy, 46 underwent distal gastrectomy with Billroth I anastomosis, and 37 with Billroth II anastomosis. The nutritional status assessment included body weight, body mass index, serum albumin, serum hemoglobin, vitamin B12, ferritin, and Nutritional Risk Index (NRI).

Results: Patients who underwent total gastrectomy had lower hemoglobin and vitamin B12 levels compared to patients who underwent distal gastrectomy. The NRI was statistically significantly lower in patients who underwent total gastrectomy than patients who underwent distal gastrectomy. Patients who underwent total gastrectomy had lower vitamin B12 than patients who underwent proximal gastrectomy till the second year after gastrectomy. Regarding postdistal gastrectomy reconstruction, there was no statistically significant difference between the Billroth I and Billroth II groups.

Conclusion: This study showed that patients who underwent total gastrectomy were more susceptible to malnutrition than those who underwent distal or proximal gastrectomy. Patients who undergo gastrectomy should be monitored carefully for nutritional status and given appropriate nutritional support in the post-operative period.

Keywords: Stomach; Stomach neoplasms; Gastrectomy; Reconstructive surgical procedures; Nutritional status

\section{INTRODUCTION}

Although deaths due to gastric cancer are decreasing [1], gastric cancer still has the second-highest incidence among all cancers in Korea and is the fourth most common cause of cancer-related deaths $[2,3]$. Recently, the diagnosis rate of early gastric cancer is gradually increasing. The 5 -year survival rate of early gastric cancer is over $90 \%[2,3]$. Due to the high incidence and improved survival rate, interest in nutritional status and quality of life in gastric cancer patients

Received September 13, 2021; Revised October 31, 2021; Accepted November 2, 2021

Corresponding author: Moon Soo Lee

E-mail msslee@schmc.ac.kr (iD ORCID https://orcid.org/0000-0002-3955-1331

(c) (1) T) This is an Open Access article distributed under the terms of the Creative Commons Attribution Non-Commercial License (http://creativecommons.org/licenses/ Copyright (c) The Korean Society of Surgical Metabolism and Nutrition and The Korean Society for Parenteral and Enteral Nutrition 
gradually increases.

The treatment of gastric cancer is radical resection, and the extent of resection varies depending on the location of gastric cancer. Typical examples are subtotal gastrectomy for lower gastric cancer, total gastrectomy or proximal gastrectomy for upper gastric cancer. Since the stomach has the function of digesting and absorbing food, when gastrectomy is performed, malnutrition may occur and the degree of malnutrition may vary depending on the extent of gastrectomy [4]. Body weight loss and anemia are representative complications caused by nutritional disorders after gastrectomy, and among them, anemia is caused by a deficiency of ferritin or vitamin B12 [5,6]. In cancer patients, anemia is associated with a decrease in quality of life and may impair the effectiveness of chemotherapy after surgery, which may eventually worsen the outcome of treatment $[4,7,8]$.

In this study, clinical, nutritional, and hemodynamic parameters were investigated before and after surgery in patients who underwent radical resection for gastric cancer, and their differences. Comparing the range of gastrectomy and the difference according to the extent of gastrectomy and the reconstructive procedure was intended to help determine an appropriate gastrectomy and reconstructive procedure to minimize nutritional disorders and find ways to improve nutrition after surgery.

\section{MATERIALS AND METHODS}

\section{Materials}

We retrospectively compared and analyzed medical records of patients who underwent radical resection for gastric cancer from December 2012 to December 2013 at Soonchunhyang University Cheonan Hospital. In a total of 107 patients, 100 patients were analyzed, excluding 2 patients who died within 1 year after surgery, 4 patients who lost followup within 1 year, and 1 patient who had cancerous bleeding before surgery. These were classified into the group that underwent distal gastrectomy, the group that underwent total gastrectomy, and the group that underwent proximal gastrectomy. The group that underwent distal gastrectomy was further divided into a group that underwent gastro-duodenal anastomosis (Billroth I) and a group that underwent gastrojejunal anastomosis (Billroth II), and each group was compared and analyzed. Proximal gastrectomy was performed on patients diagnosed with upper early gastric cancer, and esophageal-gastric anastomosis was performed as a reconstructive procedure. All groups that underwent total gastrectomy underwent Uncut Roux reconstruction.

\section{Data}

The age, sex, and pathological stage (TNM classification) of the patients were investigated. Nutritional and hemodynamic parameters include patient weight, body mass index (BMI), hemoglobin $(\mathrm{g} / \mathrm{dL})$, vitamin B12 $(\mu \mathrm{g} / \mathrm{L})$, ferritin $(\mathrm{ng} / \mathrm{mL})$, and albumin $(\mathrm{g} / \mathrm{dL})$, and nutritional risk index (NRI) were collected before surgery and $1 \sim 3$ years after surgery.

\section{Nutritional risk index}

The NRI was calculated using the following formula: $\mathrm{NRI}=(1.519 \times$ serum albumin, $\mathrm{g} / \mathrm{dL})+0.417 \times($ present body weight/ideal body weight $\times 100$ ). The ideal body weight was calculated as follows by the Lorenz formula: male, height (cm)-100-[(Height (cm)-150)/4]; female, height (cm)-100[(Height (cm)-150)/2.5].

NRI was first developed by Buzby and is widely used as a tool for simple nutritional evaluation using the ratio of blood albumin and actual body weight to usual body weight $[9,10]$. Usual body weight is generally defined as the weight maintained at least 6 months before the onset of the disease. However, since it is difficult to estimate in elderly patients or cases of underlying disease accurately, it can be replaced with an ideal body weight that can be calculated relatively simply. In this study, the ideal body weight was calculated using the Lorenz formula. When it was over 100, it was classified as normal without malnutrition (severe malnutrition: $\sim 83.5$, moderate malnutrition: 83.5 97.5, mild malnutrition: $97.5 \sim 100)$. When the current body weight was greater than the ideal body weight, the ratio between the two variables was defined as 1 .

Table 1. Patients characteristics

\begin{tabular}{lcr}
\hline \multirow{2}{*}{ Variable } & \multicolumn{2}{c}{ Statistics (n=100) } \\
\cline { 2 - 3 } Age (yr) & M SD or n (\%) & \multicolumn{1}{c}{ Median (IQR) } \\
BMI $\left(\mathrm{kg} / \mathrm{m}^{2}\right)$ & $23.19 \pm 2.58$ & $23.34(16.42 \sim 28.96)$ \\
Sex & $71(71.00)$ \\
Male & $29(29.00)$ \\
Female & \\
Type of operation & $46(46.00)$ \\
Billroth I & $37(37.00)$ \\
Billroth II & $12(12.00)$ \\
Total gastrectomy & $5(5.00)$ \\
Proximal gastrectomy & \\
TNM stage & $67(67.00)$ \\
IA & $10(10.00)$ \\
IB & $7(7.00)$ \\
IIA & $2(2.00)$ \\
IIB & $5(5.00)$ \\
IIIA & $7(7.00)$ \\
IIIB & $1(1.00)$ \\
IIIC & $1(1.00)$ \\
IV &
\end{tabular}

$\mathrm{IQR}=$ interquartile range; $\mathrm{BMI}=$ body mass index. 
Table 2. Comparison of outcomes according to extent of gastrectomy

\begin{tabular}{|c|c|c|c|c|}
\hline Variable & Preoperative & $1 \mathrm{yr}$ & $2 \mathrm{yr}$ & $3 \mathrm{yr}$ \\
\hline \multicolumn{5}{|l|}{ Hemoglobin (g/dL) } \\
\hline DG & $13.80(12.80 \sim 14.50)$ & $13.30(12.30 \sim 14.25)$ & $14.25(13.02 \sim 14.90)$ & $13.75(12.60 \sim 14.72)$ \\
\hline TG & $12.75(12.25 \sim 13.97)$ & $11.60(11.15 \sim 13.20)$ & $12.95(11.57 \sim 13.92)$ & $12.05(11.57 \sim 13.37)$ \\
\hline PG & $14.00(13.50 \sim 14.90)$ & $14.00(12.60 \sim 14.50)$ & $13.20(12.30 \sim 14.60)$ & $14.30(12.70 \sim 14.50)$ \\
\hline P-value (DG vs. TG) & 0.162 & 0.010 & 0.040 & 0.021 \\
\hline P-value (TG vs. PG) & 0.126 & 0.059 & 0.425 & 0.107 \\
\hline \multicolumn{5}{|l|}{ Ferritin (ng/mL) } \\
\hline DG & NA & $56.47(33.25 \sim 114.30)$ & $42.25(27.85 \sim 88.52)$ & $51.20(32.30 \sim 77.50)$ \\
\hline TG & NA & $63.00(48.52 \sim 136.85)$ & $34.45(27.60 \sim 41.72)$ & $53.55(36.87 \sim 76.67)$ \\
\hline PG & NA & $102.70(80.50 \sim 106.90)$ & $65.10(51.11 \sim 102.70)$ & $60.00(27.90 \sim 87.70)$ \\
\hline P-value (DG vs. TG) & NA & 0.251 & 0.260 & 0.282 \\
\hline P-value (TG vs. PG) & NA & 0.777 & 0.066 & 0.569 \\
\hline \multicolumn{5}{|l|}{ Vitamin B12 $(\mu \mathrm{g} / \mathrm{L})$} \\
\hline DG & NA & $557.45(431.70 \sim 740.70)$ & $511.75(402.87 \sim 719.32)$ & $545.60(387.80 \sim 711.17)$ \\
\hline TG & NA & $331.70(277.65 \sim 458.45)$ & $240.10(186.30 \sim 315.20)$ & 169.65 (163.95 369.55) \\
\hline PG & NA & $530.40(513.35 \sim 898.20)$ & $578.00(550.60 \sim 871.80)$ & $578.00(423.10 \sim 613.30)$ \\
\hline P-value (DG vs. TG) & NA & 0.001 & $<0.001$ & 0.007 \\
\hline P-value (TG vs. PG) & NA & 0.014 & 0.007 & 0.061 \\
\hline \multicolumn{5}{|l|}{ Albumin (g/dL) } \\
\hline DG & $4.30(4.00 \sim 4.60)$ & $4.40(4.10 \sim 4.50)$ & $4.35(4.10 \sim 4.50)$ & $4.40(4.10 \sim 4.40)$ \\
\hline TG & 4.35 (3.97 4.70) & $4.35(4.00 \sim 4.45)$ & $4.00(3.90 \sim 4.27)$ & $4.15(3.90 \sim 4.20)$ \\
\hline PG & $4.40(4.30 \sim 4.40)$ & $4.20(3.90 \sim 4.60)$ & $4.20(4.10 \sim 4.60)$ & $4.40(4.20 \sim 4.55)$ \\
\hline P-value (DG vs. TG) & 0.928 & 0.378 & 0.053 & 0.051 \\
\hline P-value (TG vs. PG) & 0.558 & 0.776 & 0.239 & 0.203 \\
\hline \multicolumn{5}{|l|}{$\operatorname{BMI}\left(\mathrm{kg} / \mathrm{m}^{2}\right)$} \\
\hline DG & $23.30(21.45 \sim 24.40)$ & $21.46(20.04 \sim 23.50)$ & 21.67 (20.61 23.77) & $21.75(20.43 \sim 24.00)$ \\
\hline TG & $24.27(22.52 \sim 24.90)$ & $19.54(19.10 \sim 20.87)$ & $20.85(20.81 \sim 21.77)$ & $21.13(21.10 \sim 21.16)$ \\
\hline PG & $25.18(21.75 \sim 26.93)$ & 20.55 (19.41 21.79) & $20.51(18.98 \sim 22.24)$ & $19.41(18.03 \sim 21.59)$ \\
\hline P-value (DG vs. TG) & 0.226 & 0.064 & 0.445 & 0.692 \\
\hline P-value (TG vs. PG) & 0.460 & 0.610 & 0.601 & 0.354 \\
\hline \multicolumn{5}{|l|}{ NRI $\left(\mathrm{kg} / \mathrm{m}^{2}\right)$} \\
\hline DG & NA & $106.13(103.03 \sim 109.05)$ & $105.88(101.74 \sim 110.46)$ & $105.93(101.85 \sim 108.24)$ \\
\hline TG & NA & $100.54(94.26 \sim 100.86)$ & 98.96 (89.79 106.37) & NA \\
\hline PG & NA & $100.03(92.89 \sim 105.91)$ & 99.51 (99.17 101.64) & $103.25(95.80 \sim 106.96)$ \\
\hline P-value (DG vs. TG) & NA & 0.003 & 0.037 & 0.539 \\
\hline P-value (TG vs. PG) & NA & 0.999 & 0.754 & NA \\
\hline \multicolumn{5}{|l|}{ Modified NRI $\left(\mathrm{kg} / \mathrm{m}^{2}\right)$} \\
\hline DG & $105.97(101.43 \sim 110.05)$ & $105.72(101.75 \sim 109.37)$ & $105.80(102.06 \sim 110.05)$ & 106.59 (101.92 108.53) \\
\hline TG & $107.01(100.82 \sim 113.09)$ & $101.85(95.52 \sim 105.20)$ & $100.94(99.11 \sim 110.11)$ & $98.04(93.22 \sim 102.86)$ \\
\hline PG & $107.11(106.71 \sim 108.53)$ & $102.72(99.25 \sim 105.40)$ & 103.97 (102.75 105.05) & $102.53(96.42 \sim 106.43)$ \\
\hline P-value (DG vs. TG) & 0.645 & 0.086 & 0.476 & 0.390 \\
\hline P-value (TG vs. PG) & 0.671 & 0.734 & 0.754 & NA \\
\hline
\end{tabular}

Values are presented as median (interquartile range).

$\mathrm{DG}$ = distal gastrectomy; $\mathrm{TG}$ = total gastrectomy; $\mathrm{PG}=$ proximal gastrectomy; $\mathrm{NA}=$ not applicable; $\mathrm{BMI}=\mathrm{body} \mathrm{max}$ index; $\mathrm{NRI}=$ nutritional risk index. 


\section{Analysis method and statistical technique}

A retrospective comparative analysis was performed based on the collected data. For continuous variables, the P-value was calculated with the Mann-Whitney U-test or the independent sample t-test. The result was presented as the mean \pm standard deviation or the median (1st quartile $\sim 3$ rd quartile). A P-value less than 0.05 was defined as statistically significant, and for statistical analysis, an Excel-based statistical analysis program, Rex (Version 3.0.3; RexSoft Co. Ltd., Seoul, Korea), was used.

This study was approved by the Institutional Review Board (IRB) of Soonchunhyang University Cheonan Hospital (IRB file number: SCHCA 2021-01-015). Written informed consent was obtained from the patients to report and publish individual patient data.

\section{RESULTS}

\section{Clinicopathological characteristics of the patients}

Among 100 patients, 71 were male, and 29 were female, the mean age was $61.46 \pm 12.54$ years, and the mean BMI was $23.19 \pm 2.58 \mathrm{~kg} / \mathrm{m}^{2} .12$ patients underwent total gas- trectomy, 5 patients underwent proximal gastrectomy, and 83 patients underwent distal gastrectomy. Reconstruction after total gastrectomy was performed using the Uncut Roux procedure. For reconstruction after proximal gastrectomy, esophago-gastric anastomosis was performed. Of the patients who underwent distal gastrectomy, 46 underwent gastroduodenal anastomosis, and 37 underwent gastro-jejunal anastomosis. According to the American Joint Committee on Cancer 7 th edition, pathological stage (TNM stage) was 77 patients in stage 1, 9 in stage 2, 13 in stage 3, and 1 in stage 4 (Table 1).

\section{Comparison according to the extent of gastrectomy}

Hemoglobin level, ferritin, vitamin B12, albumin, BMI, and NRI were compared and analyzed between the two groups and 1 3 years after surgery for distal gastrectomy and total gastrectomy. In addition, the same variables were compared and analyzed for proximal gastrectomy and total gastrectomy.

There was no statistically significant difference in the preoperative indicators in the comparison between distal gastrectomy and total gastrectomy. The hemoglobin level and vitamin B12 were compared to the distal gastrectomy group
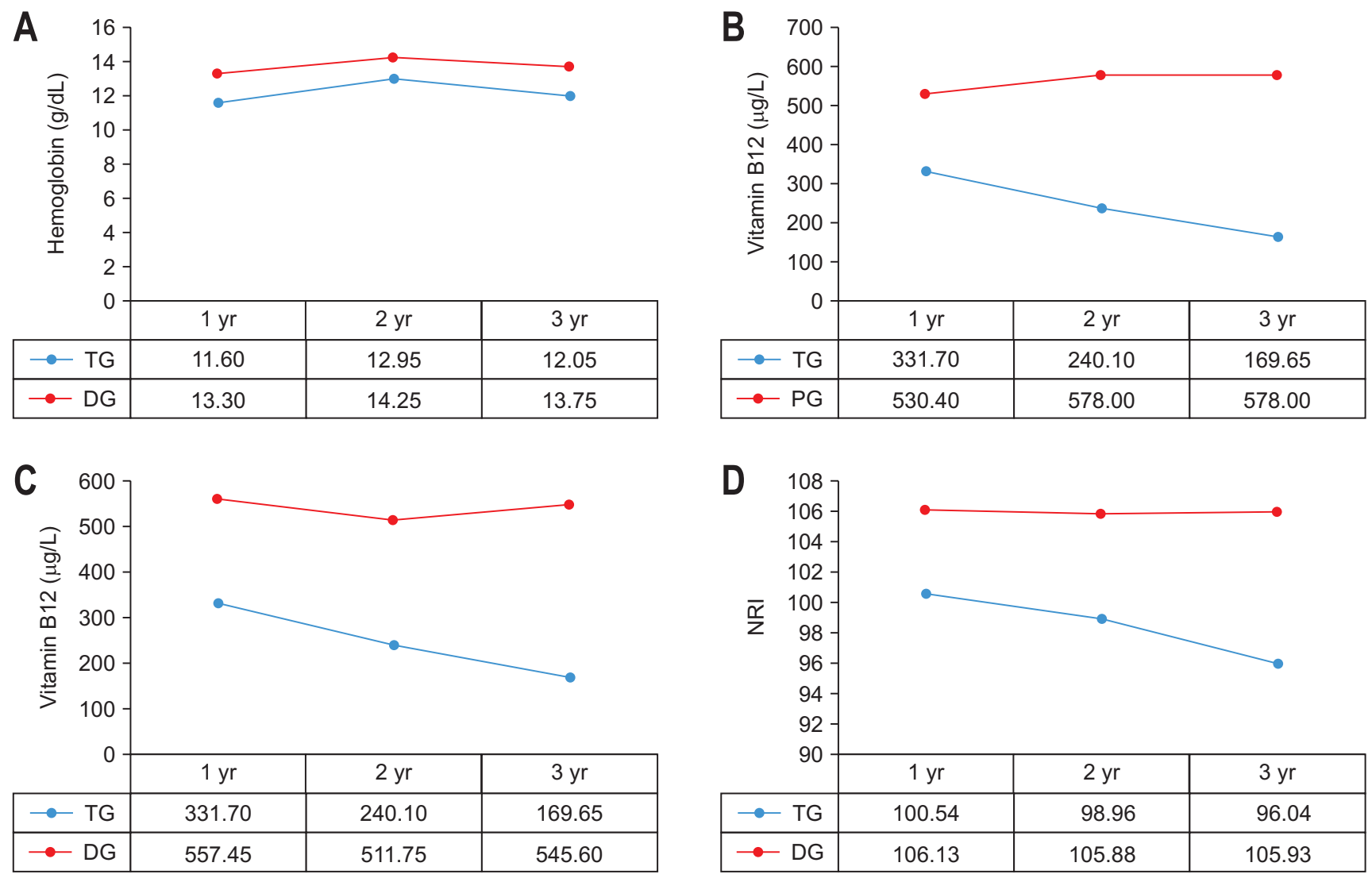

Fig. 1. Comparison of outcomes according to extent of gastrectomy. (A) Comparison of Hb between TG and DG. (B) Comparison of vitamin B12 between TG and PG. (C) Comparison of vitamin B12 between TG and DG. (D) Comparison of NRI between TG and DG. $\mathrm{TG}=$ total gastrectomy; $\mathrm{DG}=$ distal gastrectomy; $\mathrm{PG}=$ proximal gastrectomy; $\mathrm{NRI}$ = nutritional risk index. 
in the total gastrectomy group. It was statistically significantly lower during the 3-year period (P-values of $1 \sim 3$ years after surgery $=0.010,0.040,0.021)$. There was no significant difference between the two groups in the NRI using the ideal body weight. The NRI was measured by assuming that the body weight measured before surgery was the usual body weight was statistically significantly lower at 1 year and 2 years after surgery in the group that underwent total gastrec- tomy (P-values of $1 \sim 2$ years after surgery $=0.003,0.037$ ), and there was no difference at 3 years after surgery. Vitamin B12 was significantly higher in the group that underwent proximal gastrectomy up to 2 years after surgery than in the group that underwent total gastrectomy. However, there was no significant difference between the two groups after 3 years after surgery (P-values of $1 \sim 2$ years after surgery $=0.014$, 0.007) (Table 2, Fig. 1).

Table 3. Comparison of outcomes according to reconstruction method in distal gastrectomy group

\begin{tabular}{|c|c|c|c|}
\hline Variable & Billroth I & Billroth II & P-value \\
\hline \multicolumn{4}{|c|}{ Hemoglobin (g/dL) } \\
\hline Preoperative & $13.75(12.60 \sim 14.55)$ & $13.80(12.90 \sim 14.50)$ & 0.518 \\
\hline $1 \mathrm{yr}$ & $12.65(11.57 \sim 13.85)$ & $12.70(11.70 \sim 14.20)$ & 0.497 \\
\hline $2 \mathrm{yr}$ & $14.10(13.00 \sim 14.65)$ & $14.35(13.40 \sim 15.20)$ & 0.215 \\
\hline $3 \mathrm{yr}$ & $13.75(12.55 \sim 14.65)$ & $14.40(13.40 \sim 15.40)$ & 0.099 \\
\hline \multicolumn{4}{|l|}{ Ferritin (ng/mL) } \\
\hline Preoperative & NA & NA & \\
\hline $1 \mathrm{yr}$ & $69.25(41.42 \sim 121.87)$ & $46.10(28.30 \sim 77.30)$ & 0.073 \\
\hline $2 \mathrm{yr}$ & $59.25(34.53 \sim 106.07)$ & $34.05(24.37 \sim 55.22)$ & 0.065 \\
\hline $3 \mathrm{yr}$ & 47.80 (33.35 67.30) & $54.20(31.30 \sim 76.45)$ & 0.922 \\
\hline \multicolumn{4}{|c|}{$\operatorname{Vitamin} \mathrm{B} 12(\mu \mathrm{g} / \mathrm{L})$} \\
\hline Preoperative & NA & NA & \\
\hline $1 \mathrm{yr}$ & $548.20(444.75 \sim 713.57)$ & $571.70(423.60 \sim 758.50)$ & 0.989 \\
\hline $2 \mathrm{yr}$ & $522.40(404.15 \sim 680.20)$ & $589.50(377.20 \sim 761.70)$ & 0.799 \\
\hline $3 \mathrm{yr}$ & $521.55(391.80 \sim 822.25)$ & $532.25(361.02 \sim 657.30)$ & 0.438 \\
\hline \multicolumn{4}{|l|}{$\operatorname{Albumin}(\mathrm{g} / \mathrm{dL})$} \\
\hline Preoperative & $4.40(4.02 \sim 4.65)$ & $4.35(4.00 \sim 4.65)$ & 0.733 \\
\hline $1 \mathrm{yr}$ & $4.30(4.10 \sim 4.50)$ & $4.40(4.10 \sim 4.50)$ & 0.596 \\
\hline $2 \mathrm{yr}$ & $4.30(4.10 \sim 4.50)$ & $4.40(4.20 \sim 4.60)$ & 0.400 \\
\hline $3 \mathrm{yr}$ & $4.35(4.02 \sim 4.47)$ & $4.40(4.20 \sim 4.40)$ & 0.297 \\
\hline \multicolumn{4}{|l|}{$\mathrm{BMI}\left(\mathrm{kg} / \mathrm{m}^{2}\right)$} \\
\hline Preoperative & $23.29(21.53 \sim 24.55)$ & 23.38 (21.32 24.28) & 0.872 \\
\hline $1 \mathrm{yr}$ & $21.35(20.56 \sim 22.81)$ & 21.96 (19.60 24.08) & 0.612 \\
\hline $2 \mathrm{yr}$ & 21.53 (20.67 22.79) & 21.67 (20.61 23.93) & 0.606 \\
\hline $3 \mathrm{yr}$ & $21.53(20.27 \sim 23.87)$ & $22.89(20.52 \sim 24.06)$ & 0.537 \\
\hline \multicolumn{4}{|l|}{ NRI } \\
\hline Preoperative & NA & NA & \\
\hline $1 \mathrm{yr}$ & $106.51(104.95 \sim 109.06)$ & $104.84(102.57 \sim 108.43)$ & 0.227 \\
\hline $2 \mathrm{yr}$ & 106.17 (100.05 109.38) & $105.88(102.38 \sim 110.75)$ & 0.281 \\
\hline $3 \mathrm{yr}$ & $105.63(99.10 \sim 107.96)$ & $105.94(104.49 \sim 108.24)$ & 0.377 \\
\hline \multicolumn{4}{|l|}{ Modified NRI } \\
\hline Preoperative & $106.29(101.82 \sim 111.19)$ & $105.49(100.94 \sim 110.05)$ & 0.454 \\
\hline $1 \mathrm{yr}$ & $106.70(102.31 \sim 109.76)$ & $105.23(101.28 \sim 108.53)$ & 0.398 \\
\hline $2 \mathrm{yr}$ & $105.64(101.06 \sim 109.93)$ & $106.45(102.46 \sim 110.05)$ & 0.376 \\
\hline $3 \mathrm{yr}$ & $107.01(100.36 \sim 108.53)$ & $106.32(103.91 \sim 108.53)$ & 0.861 \\
\hline
\end{tabular}

Values are presented as median (interquartile range).

$\mathrm{NA}=$ not applicable; $\mathrm{BMI}=$ body max index; NRI = nutritional risk index. 

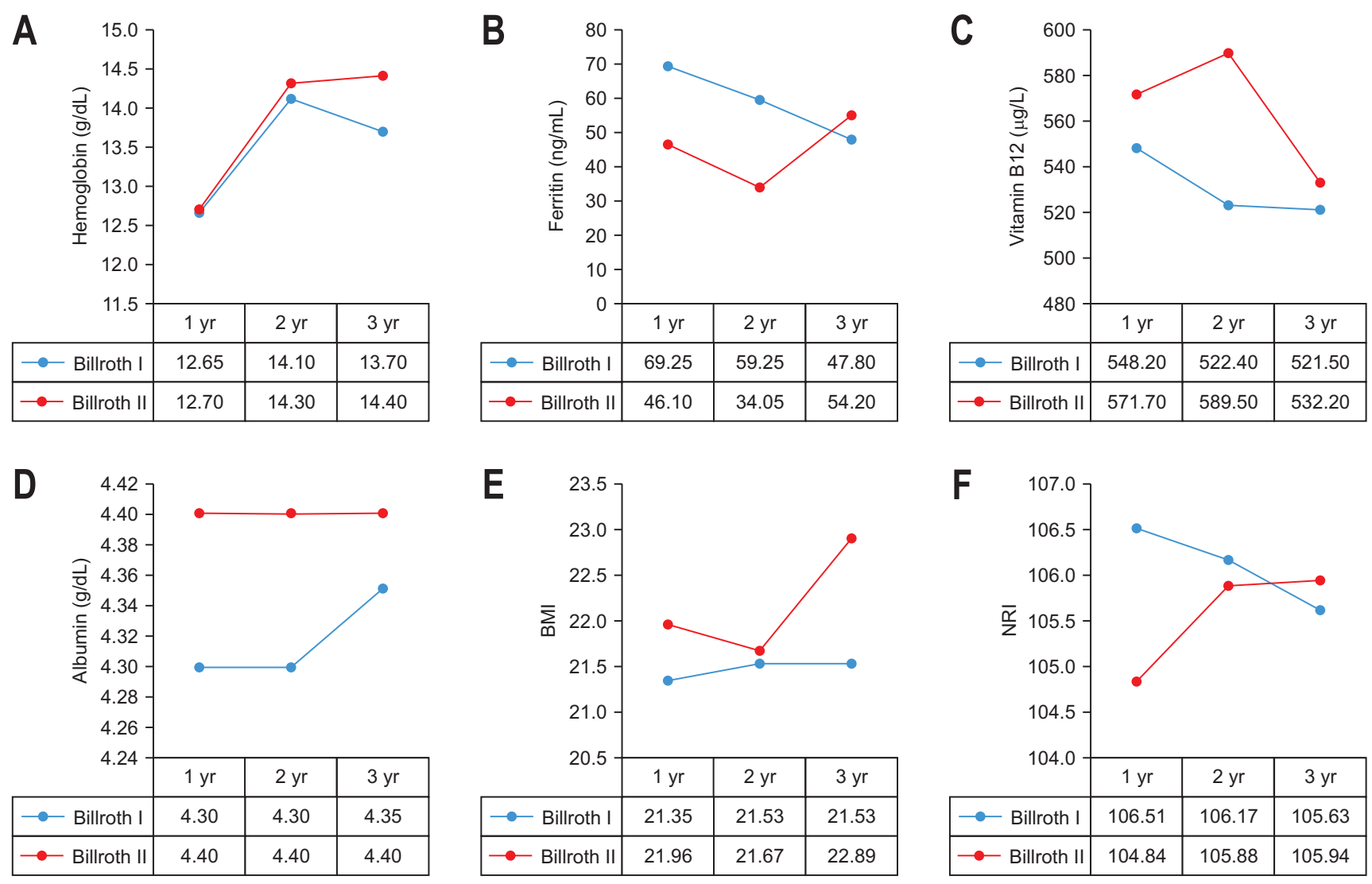

Fig. 2. Comparison of outcomes according to reconstruction method in distal gastrectomy group. (A) Comparison of $\mathrm{Hb}$ between Billoth I and Billoth II. (B) Comparison of Ferritin between Billoth I and Billoth II. (C) Comparison of vitamin B12 between Billoth I and Billoth II. (D) Comparison of albumin between Billoth I and Billoth II. (E) Comparison of BMI between Billoth I and Billoth II. (F) Comparison of NRI between Billoth I and Billoth II.

$\mathrm{BMI}=$ body mass index; $\mathrm{NRI}=$ nutritional risk index.

\section{Comparison according to reconstruction method}

In the group that underwent distal gastrectomy, the group underwent gastro-duodenal anastomosis, and the group that underwent gastro-jejunal anastomosis according to the reconstruction procedure. The NRI was compared and analyzed before surgery and for 1 3 years after surgery. There was no statistically significant difference between the two groups in the pre-operative indices. There was no statistically significant difference in the post-operative indices between the two groups. The ferritin level was higher in the group with gastro-duodenal anastomosis at 1 2 years after surgery, but it was not statistically significant (median for each group, 1 year post-surgery: $69.25 \mathrm{ng} / \mathrm{mL}$ vs. $46.10 \mathrm{ng} / \mathrm{mL}$, Pvalue $=0.073,2$ years post-surgery: $59.25 \mathrm{ng} / \mathrm{mL}$ vs. 34.05 $\mathrm{ng} / \mathrm{mL}, \mathrm{P}$-value $=0.065)$ (Table 3, Fig. 2).

\section{DISCUSSION}

Various nutritional changes can occur after gastrectomy, and malnutrition after gastrectomy is a factor related to poor prognosis after surgery in gastric cancer patients [11]. Recently, interest in the quality of life after surgery has increased as the long-term survival rate has increased due to early diagnosis and improvement in surgical outcomes in gastric cancer. For this reason, studies on nutritional changes that may occur after gastrectomy are being actively conducted. Previously, in evaluating and comparing the extent of resection or reconstruction type in gastric cancer patients, the focus was on safety from an oncological point of view and fewer complications directly related to the surgical procedure. On the other hand, relatively few studies have compared the difference between the extent of resection and the reconstruction type from a nutritional point of view. Furthermore, malnutrition increases postoperative complications and is known to increase the risk of postoperative infection as it causes immunodeficiency in particular [11]. This also lengthens hospital stay, which is why screening and correction of malnutrition are important in cost-effectiveness.

One of the most common nutritional complications after gastrectomy is anemia. It is mainly caused by a deficiency 
of ferritin and vitamin B12 after gastrectomy $[4,6,7]$. It is known that ferritin deficiency after gastrectomy is influenced by decreased gastric acid secretion, duodenum and upper jejunum bypass, and rapid intestinal transit $[7,12]$. Vitamin B12 deficiency is due to a decrease in intrinsic factor, which is secreted in parietal cells of the stomach. It is known to play an important role in the absorption of vitamin B12 [13]. It is said that anemia occurs in about $18 \%$ of patients who have undergone radical resection for gastric cancer [12]. In a large cohort study, anemia occurred in $18.7 \%$ of patients 1 year after surgery and $39.5 \%$ within 5 years after surgery [7]. Regarding the extent of resection, it is known that total gastric resection has a higher risk of anemia than partial resection [4]. In this study, hemoglobin levels and vitamin B12 levels were significantly lower in the total resection group than in the partial resection group. Since it is known that the maximum absorption of ferritin occurs in the duodenum and the upper jejunum, it is theoretically possible to develop anemia in the gastro-duodenal anastomosis, considering that the bypass mechanism causes the loss of the mucosal surface necessary for the absorption of ferritin and contributes to the development of anemia. Although this is expected to occur low [14], reports show no significant difference between gastro-duodenal anastomosis and gastro-jejunal anastomosis after partial resection [4]. Studies showing differences between the two groups were rare, and most did not show a significant difference. There was no statistically significant difference between the two groups in this study. However, although it was not statistically significant in this study, ferritin levels were higher in the group undergoing gastro-duodenal anastomosis until $1 \sim 2$ years after surgery. Considering the small population, additional validation through follow-up studies is likely to be necessary, and additional study through long-term follow-up is needed for early gastric cancer patients who are expected to survive long-term for other variables except for ferritin. In a study on iron and vitamin B12 supplementation for the prevention or treatment of anemia, a recent prospective randomized clinical trial demonstrated that iron supplementation via the intravenous route was effective in postoperative anemia patients with gastric cancer [15-17]. In addition, several studies have reported that oral administration of vitamin B12 is effective in gastrectomy patients $[13,18]$. Therefore in total gastrectomy patients, who are more susceptible to malnutrition, we can prevent postoperative anemia in this pathway. Additional studies are needed to establish an additional iron and vitamin B12 supplementation protocol according to the extent of gastrectomy and the type of reconstruction for the patient group with expected long-term survival in the future.

Various anthropometric and serological indicators are used as indicators to evaluate a patient's nutritional status. In addition, nutritional screening and diagnostic methods for more efficient evaluation have been developed and used. One of the representative examples is the NRI. The NRI is a useful nutritional screening index that can be easily calculated using objective data such as body weight and albumin. It was designed to predict surgical complications in malnourished patients and is used as an index to evaluate nutritional status in head and neck cancer and gastrointestinal cancer $[9,10]$. A previous report showed that the NRI was significantly lower in gastric cancer patients who underwent total gastrectomy [8]. This study confirmed that the NRI was lower in the group that underwent total gastrectomy than in the group that underwent distal gastrectomy up to 2 years after surgery. Therefore, it is necessary to continuously check and manage their nutritional status in patients who underwent total gastrectomy. To overcome this, various studies are being conducted to increase the amount of food by administering oral ghrelin to the patient group who underwent total gastrectomy [19].

Among 9 and 13 patients with stage 2 and 3, both 8 patients were treated with chemotherapy. Independent of surgical method, considering that chemotherapy itself can induce anemia in patients through myelosuppressive mechanism, comparison of this is necessary. A study of relationship between chemotherapy and anemia reported that $40 \%$ of patients receiving chemotherapy developed anemia, and of these $68 \%$ of gastric cancer patients developed anemia [20]. In this study, similarly, in the case of stage 2 and 3 patients, anemia was more common in chemotherapy performed patients than whom do not performed chemotherapy.

The results of this study confirmed that there was a significant difference in nutrition between total gastrectomy and distal gastrectomy. However, from a nutritional point of view, there was no statistically significant difference from proximal gastrectomy, which is a partial gastrectomy for upper gastric cancer patients. Furthermore, in a comparative study of postoperative outcomes through long-term follow-up between the two groups, there were no differences in oncological outcomes or postoperative complications. Considering that the group that underwent proximal gastrectomy showed a less decrease in hemoglobin level, less weight loss, and less incidence of severe gastroesophageal reflux compared to the group that underwent total gastrectomy [21], there was likely no difference due to small patient numbers in this study, and needs to be supplemented through follow-up studies.

\section{CONCLUSION}

The results of this study show that nutritional status can change after gastrectomy, and the degree of change is affected by the extent of gastrectomy. However, given that this study is a retrospective study, it is a single-institution study, the patient number is small, and the follow-up period is relatively short, there is a limit to clearly showing differences in nutritional status according to the type of surgery with the results of this study. Therefore, to supplement this, a longterm, large-scale study should be conducted on the extent of resection and the differences in nutritional status according 
to the reconstruction method. As these studies progress, they may provide additional information and guideline in determining the extent of resection and reconstructive surgery. In addition, it will be helpful to develop individual nutritional status management guidelines tailored to each surgical procedure by analyzing changes in nutritional status according to the types of resection and reconstruction.

\section{AUTHOR CONTRIBUTIONS}

Conceptualization: KHK, JHY. Data curation: KHK, JHY. Formal analysis: GJS, MWS. Funding acquisition: KHK, JHY. Investigation: KHK, JHY. Methodology: GJS, MWS. Project administration: KHK, JHY. Resources: GJS, MWS. Software: JHY, GJS. Supervision: SYK, MSL. Validation: MJB, MSL. Visualization: MJB, MSL. Writing - original draft: KHK, JHY. Writing - review \& editing: SYK, MJB, MSL.

\section{CONFLICTS OF INTEREST}

The authors of this manuscript have no conflicts of interest to disclose.

\section{ORCID}

Kyung Hwan Kim, https://orcid.org/0000-0002-8420-3782 Jong Hyuk Yoon, https://orcid.org/0000-0002-8746-4945 Geum Jong Song, https://orcid.org/0000-0002-1067-8099 Myoung Won Son, https://orcid.org/0000-0001-5255-9234 Sung Yong Kim, https://orcid.org/0000-0002-2070-0915 Moo Jun Baek, https://orcid.org/0000-0003-3567-6687 Moon Soo Lee, https://orcid.org/0000-0002-3955-1331

\section{REFERENCES}

1. Bray F, Ferlay J, Soerjomataram I, Siegel RL, Torre LA, Jemal A. Global cancer statistics 2018: GLOBOCAN estimates of incidence and mortality worldwide for 36 cancers in 185 countries. CA Cancer J Clin 2018;68:394-424.

2. Shin A, Kim J, Park S. Gastric cancer epidemiology in Korea. J Gastric Cancer 2011;11:135-40.

3. Information Committee of Korean Gastric Cancer Association. Korean Gastric Cancer Association nationwide survey on gastric cancer in 2014. J Gastric Cancer 2016;16:13140.

4. Hong S, Won YJ, Park YR, Jung KW, Kong HJ, Lee ES; Community of Population-Based Regional Cancer Registries. Cancer statistics in Korea: incidence, mortality, survival, and prevalence in 2017. Cancer Res Treat 2020;52:33550.

5. Lim CH, Kim SW, Kim WC, Kim JS, Cho YK, Park JM, et al. Anemia after gastrectomy for early gastric cancer: longterm follow-up observational study. World J Gastroenterol 2012;18:6114-9.

6. Beyan C, Beyan E, Kaptan K, Ifran A, Uzar Al. Post-gastrec- tomy anemia: evaluation of 72 cases with post-gastrectomy anemia. Hematology 2007;12:81-4.

7. Jun JH, Yoo JE, Lee JA, Kim YS, Sunwoo S, Kim BS, et al. Anemia after gastrectomy in long-term survivors of gastric cancer: a retrospective cohort study. Int J Surg 2016;28:162-8.

8. Kim KH, Park DJ, Park YS, Ahn SH, Park DJ, Kim HH. Actual 5-year nutritional outcomes of patients with gastric cancer. J Gastric Cancer 2017;17:99-109.

9. Barge-Caballero E, García-López F, Marzoa-Rivas R, BargeCaballero G, Couto-Mallón D, Paniagua-Martín MJ, et al. Prognostic value of the nutritional risk index in heart transplant recipients. Rev Esp Cardiol (Engl Ed) 2017;70:63945.

10. Pokharel N, Katwal G, Adhikari SK. Comparison of preoperative Nutritional Risk Index and Body Mass Index for predicting immediate postoperative outcomes following major gastrointestinal surgery: cohort-study. Ann Med Surg (Lond) 2019;48:53-8.

11. Fujiya K, Kawamura T, Omae K, Makuuchi R, Irino T, Tokunaga $M$, et al. Impact of malnutrition after gastrectomy for gastric cancer on long-term survival. Ann Surg Oncol 2018;25:974-83.

12. Kim JH, Bae YJ, Jun KH, Chin HM. The prevalence and clinical significance of postgastrectomy anemia in patients with early-stage gastric cancer: a retrospective cohort study. Int J Surg 2018;52:61-6.

13. Moleiro J, Mão de Ferro S, Ferreira S, Serrano M, Silveira M, Dias Pereira A. Efficacy of long-term oral vitamin B12 supplementation after total gastrectomy: results from a prospective study. GE Port J Gastroenterol 2018;25:11722.

14. Lee JH, Hyung WJ, Kim HI, Kim YM, Son T, Okumura N, et al. Method of reconstruction governs iron metabolism after gastrectomy for patients with gastric cancer. Ann Surg 2013;258:964-9.

15. Reim D, Kim YW, Nam BH, Kim MJ, Yook JH, Park YK, et al. FAIRY: a randomized controlled patient-blind phase III study to compare the efficacy and safety of intravenous ferric carboxymaltose (Ferinject ${ }^{\circledR}$ ) to placebo in patients with acute isovolemic anemia after gastrectomy - study protocol for a randomized controlled trial. Trials 2014;15:111.

16. Yoon HM, Kim YW, Nam BH, Reim D, Eom BW, Park JY, et al. Intravenous iron supplementation may be superior to observation in acute isovolemic anemia after gastrectomy for cancer. World J Gastroenterol 2014;20:1852-7.

17. Jeong O, Park YK. Effect of intravenous iron supplementation for acute postoperative anemia in patients undergoing gastrectomy for gastric carcinoma: a pilot study. Ann Surg Oncol 2014;21:547-52.

18. Kim HI, Hyung WJ, Song KJ, Choi SH, Kim CB, Noh SH. Oral vitamin B12 replacement: an effective treatment for vitamin B12 deficiency after total gastrectomy in gastric cancer patients. Ann Surg Oncol 2011;18:3711-7.

19. Takiguchi S, Takata A, Murakami K, Miyazaki Y, Yanagimoto Y, Kurokawa Y, et al. Clinical application of ghrelin administration for gastric cancer patients undergoing gastrectomy. Gastric Cancer 2014;17:200-5.

20. Kitano T, Tada H, Nishimura T, Teramukai S, Kanai M, 
Nishimura T, et al. Prevalence and incidence of anemia in Japanese cancer patients receiving outpatient chemotherapy. Int J Hematol 2007;86:37-41.

21. Son MW, Kim YJ, Jeong GA, Cho GS, Lee MS. Long- term outcomes of proximal gastrectomy versus total gastrectomy for upper-third gastric cancer. J Gastric Cancer $2014 ; 14: 246-51$. 